\title{
A high-throughput method for quantifying metabolically active yeast cells
}

Nandy, Subir Kumar; Knudsen, Peter Boldsen; Rosenkjær, Alexander; Eliasson Lantz, Anna; Thykær, Jette; Workman, Mhairi

Published in:

Yeast

Link to article, DOI:

10.1002/yea.3072

Publication date:

2015

Document Version

Publisher's PDF, also known as Version of record

Link back to DTU Orbit

Citation $(A P A)$ :

Nandy, S. K., Knudsen, P. B., Rosenkjær, A., Eliasson Lantz, A., Thykær, J., \& Workman, M. (2015). A highthroughput method for quantifying metabolically active yeast cells. Yeast, 32(6), 461-468.

https://doi.org/10.1002/yea.3072

\section{General rights}

Copyright and moral rights for the publications made accessible in the public portal are retained by the authors and/or other copyright owners and it is a condition of accessing publications that users recognise and abide by the legal requirements associated with these rights.

- Users may download and print one copy of any publication from the public portal for the purpose of private study or research.

- You may not further distribute the material or use it for any profit-making activity or commercial gain

- You may freely distribute the URL identifying the publication in the public portal

If you believe that this document breaches copyright please contact us providing details, and we will remove access to the work immediately and investigate your claim 


\section{S. K. Nandy et al.}

The methylene blue dye reduction test (MBRT) is widely used in the dairy industry to determine the microbial load in milk. This method relies on sample decoloration rate as a prognostic indicator for the microbial load in the sample. Although the mechanism by which methylene blue is reduced has not been entirely elucidated, it is suggested to be a combination of oxygen consumption in conjunction with production of redox equivalents and reduction directly by membrane-bound reductases (Pettipher et al., 1980; Atherton and Newlander, 1977; Learoyd et al., 1992). The MBRT technique has previously been described for bacterial and, more recently, yeast cell cultures, focusing on quantification of metabolically active bacteria and evaluation of overall yeast vitality, respectively (Bapat et al., 2006; Li et al., 2011). Although the method has been described for yeast, previous work focused solely on evaluation of culture vitality as a means of identifying when a culture is at its metabolic apex and, therefore, optimal as an inoculum for a new fermentation (Li et al., 2011). In a recent study, an absorption and desorption assay based on methylene blue was devised, proving a linear correlation between methylene blue absorption/desorption and dry weight (Fischer and Sawers, 2013); this method, however, necessitates microorganism-specific correlation coefficients. In this study we expanded the MBRT method to a high-throughput quantitative tool for the physiological characterization of yeast, with full scalability from 1 litre bioreactors to a high-throughput microtitre scale. By discriminating between metabolically active and inactive cells, this method allows for the establishment of growth rates, representing only the relevant cultivable fraction of the cultures, not attainable with standard methods. This discriminatory nature of the method further enables the detection of the viability drop at the diauxic shift and for death phase characterization, with further applications in studies where cell viability and death measurements are required, e.g. antimicrobial screening (Holm et al., 2014). Finally, the presented method offers an attractive alternative for CFU estimation compared with the traditional spread-plate technique.

\section{Materials and methods}

\section{Microorganism}

Saccharomyces cerevisiae CEN.PK113-7D (MAT $\alpha$, MAL2-8c SUC2) was applied as the model organism for this study. The strain was maintained on yeast extract-peptone-glucose agar plates. A single colony from a plate was subcultured before each test in a shake flask with $100 \mathrm{ml}$ sterile minimal medium and grown for $14-16 \mathrm{~h}$ at $30^{\circ} \mathrm{C}$ at $150 \mathrm{rpm}$. Bioreactors and microtitre plates were inoculated to $\mathrm{OD}=0.01$ and the entire submerged cultivation process carried out over $\sim 30-40 \mathrm{~h}$.

\section{Media}

Yeast extract, peptone and glucose medium (YPG) was used throughout the experiments to grow the organism. YPG agar was used for culture maintenance on plates and for viable counts, using the spread-plate method. The minimal medium composition used for cultivation in either the bioreactors and in 48-well plates was as follows: $20 \mathrm{~g} / \mathrm{l}$ glucose, $5 \mathrm{~g} / \mathrm{l}$ ammonium sulphate, $3 \mathrm{~g} / 1$ monopotassium phosphate, $0.5 \mathrm{~g} / 1$ magnesium sulphate, $1 \mathrm{ml}$ trace metal solution, $1 \mathrm{ml}$ vitamin solution, $50 \mu \mathrm{l}$ antifoam 204 (Sigma-Aldrich, St. Louis, MO, USA). The trace metal solution consisted of $15 \mathrm{~g} / \mathrm{l}$ EDTA (sodium salt), $0.45 \mathrm{~g} / 1 \mathrm{ZnSO}_{4} \cdot 7 \mathrm{H}_{2} \mathrm{O}$, $1 \mathrm{~g} / \mathrm{l} \quad \mathrm{MnCl}_{2} \cdot 2 \mathrm{H}_{2} \mathrm{O}, \quad 0.3 \mathrm{~g} / \mathrm{l} \quad \mathrm{CoCl}_{2} \cdot 6 \mathrm{H}_{2} \mathrm{O}, \quad 0.3 \mathrm{~g} / \mathrm{l}$ $\mathrm{CuSO}_{4} \cdot 5 \mathrm{H}_{2} \mathrm{O}, \quad 0.4 \mathrm{~g} / \mathrm{l} \quad \mathrm{Na}_{2} \mathrm{MoO}_{4} \cdot 2 \mathrm{H}_{2} \mathrm{O}, \quad 0.45 \mathrm{~g} / \mathrm{l}$ $\mathrm{CaCl}_{2} \cdot 2 \mathrm{H}_{2} \mathrm{O}, 0.3 \mathrm{~g} / \mathrm{l} \quad \mathrm{FeSO}_{4} \cdot 7 \mathrm{H}_{2} \mathrm{O}, 0.1 \mathrm{~g} / \mathrm{l}_{3} \mathrm{H}_{3}$ and $0.1 \mathrm{~g} / \mathrm{l} \mathrm{KI}$. The vitamin solution contained $0.05 \mathrm{~g} / \mathrm{l}$ biotin, $0.2 \mathrm{~g} / \mathrm{l} p$-amino benzoic acid, $1 \mathrm{~g} / \mathrm{l}$ nicotinic acid, $1 \mathrm{~g} / \mathrm{l} \mathrm{Ca}$-pantothenate, $1 \mathrm{~g} / \mathrm{l}$ pyridoxine$\mathrm{HCl}, 1 \mathrm{~g} / \mathrm{l}$ thiamine- $\mathrm{HCl}$ and $25 \mathrm{~g} / \mathrm{l}$ myo-inositol. For high-throughput experiments, the medium was supplemented with $100 \mathrm{mM}$ 4-morpholine-ethanosulphonic acid hemisodium salt (MES) buffer (Sigma-Aldrich) and adjusted to $\mathrm{pH} 5 ; 1 \%$ methylene blue dye (Sigma-Aldrich) was used throughout the experiments. The chemicals for YPG and minimal medium were obtained from Merck Millipore (Darmstadt, Germany) or Sigma-Aldrich.

\section{Standard calibration curve and sensitivity analysis of MBRT}

Correlation curves for optical density, viable cells and methylene blue decoloration rates were based on yeast shake-flask cultures incubated at $30^{\circ} \mathrm{C}$ under constant shaking at $150 \mathrm{rpm}$. The whole culture (about $100 \mathrm{ml}$ ) was aseptically harvested after $16 \mathrm{~h}$, while still in the exponential growth phase, and centrifuged at $17000 \times g$ for $10 \mathrm{~min}$ at $4^{\circ} \mathrm{C}$, after which serial dilutions were prepared in phosphate- 
buffered saline (Bapat et al., 2006). The sensitivity analysis, previously described by Bapat et al. (2006), was modified for the automated highthroughput set-up in 96-well plates and performed in triplicate.

In brief, serial diluted yeast samples were dispensed in aliquots of $100 \mu 1$ into a 96-well plate (Greiner Bio-One, Frickenhausen, Germany) and the optical density (OD) measured at $600 \mathrm{~nm}$. After OD measurement, $2 \mu$ methylene blue dye was automatically dispensed into the wells and the plate subsequently shaken for $5 \mathrm{~s}$ before colour reduction time was recorded by kinetic measurements at $650 \mathrm{~nm}$. For validation, three or four diluted yeast samples were applied for CFU spread-plate counts and incubated at $30^{\circ} \mathrm{C}$ for $36 \mathrm{~h}$ prior to counting.

\section{S. cerevisiae growth experiment in bioreactors}

The reference cultivations of $S$. cerevisiae were carried out in batch bioreactors with a working volume of 1 litre (Applikon Biotechnology, Schiedam, The Netherlands). Temperature was maintained at $30^{\circ}$ $\mathrm{C}$, agitation at $600 \mathrm{rpm}$ and aeration was set to 1 vvm. $\mathrm{pH} 5$ was maintained by automatic addition of $2 \mathrm{~N} \mathrm{NaOH}$; the pH electrode (Mettler Toledo, $\mathrm{OH}, \mathrm{USA}$ ) was calibrated according to the manufacturer's standard procedures. The bioreactors were equipped with a condenser to avoid evaporation from the medium. The effluent gas from the fermentation was monitored using an acoustic gas analyser (1311, Innova Air Tech Instrument A/S, Nærum, Denmark). Samples were taken at regular intervals for optical density measurement, viable plate count and MBRT. The decoloration slope for each sample was evaluated spectrophotometrically over a $200 \mathrm{~s}$ period, following the procedure outlined in Bapat et al., 2006. Experiments were conducted as three true replicates, with the average reported. The linear interval obtained from the sensitivity analysis was correlated with the MBRT to yield CFU estimates, which was validated by independent CFU counts.

\section{Standard calibration curve and sensitivity analysis of MBRT}

For high-throughput fermentations, the inoculum was prepared from overnight cultures to a final $\mathrm{OD}=0.01$. The inoculum was automatically seeded into 48-well microtitre plates (Greiner Bio-One) in aliquots of $400 \mu \mathrm{l}$, using a STAR liquid handling workstation (Hamilton, Bonaduz, Switzerland), with an integrated Thermo Cytomat 2 C450 shaking incubator (Thermo-Fischer Scientific, Waltham, MA, USA) and Biotek Synergy 2 microplate reader equipped with a dual reagent dispenser (Biotek, Winooski, VT, USA). The outer wells were filled with water to reduce the effects of evaporation on the microcultures and four inner wells were filled with medium, serving as negative references. The plates were incubated at $30^{\circ} \mathrm{C}$ in the integrated incubator under constant shaking at $1200 \mathrm{rpm}$ with an amplitude of $3 \mathrm{~mm}$. Humidity was kept constant by a heated water bath integrated into the shaking incubator. The handling workstation was programed to extract individual plates from the incubator at userdefined time intervals $(\sim 2 \mathrm{~h})$ for measurements and liquid handling. As a precaution, random samples were taken and investigated under the microscope for cross-contamination.

Briefly, plates were extracted and transported by the handling system to the plate reader for OD measurements, after which samples of $100 \mu \mathrm{l}$ from three individual wells were automatically sampled for the MBRT method. The 48-well cultivation plate was then returned to the incubator. Finally, the 96-well plates containing the samples was transferred to the plate reader, where $2 \mu$ l methylene blue dye was dispensed into the wells, using the inbuilt dual reagent dispenser. The methylene blue was mixed in by continuous shaking and reduction time was recorded using kinetic measurements at $650 \mathrm{~nm}$ over $2 \mathrm{~min}$, at $7 \mathrm{~s}$ intervals.

\section{Results and discussion}

The principal aim of this study was to introduce an efficient and inexpensive tool for quantifying metabolically active yeasts and relating this to actual OD measurements. The scalability was thoroughly challenged in bioreactor settings and microtitre plates before introduction of an automated high-throughput set-up. Through these studies, a new method was developed, broadening the range of physiological parameters that may be evaluated in automated high-throughput settings. This new tool is well suited for automated growth curve analysis and will be highly applicable for automated high-throughput antimicrobial drug screening, such as described in Holm 
et al. (2014). Towards the aim of implementing MBRT for a high-throughput set-up for $S$. cerevisiae, we focused on bioreactors as reference for the high-throughput system.

\section{Comparing OD and CFU with MBRT}

The sensitivity analysis using $S$. cerevisiae demonstrated that the MBRT-derived decoloration slope can be used to correlate the OD of metabolically active cell cultures with CFU for an order of magnitude $>1000$ cells. By adding in this step, the MBRT method times were reduced from several hours to $\sim 40-60 \mathrm{~s}$. The correlation of MBRT slope to OD and CFU (as shown in Figure 1a, b, respectively) was established to be linear and sensitive. The linearity of the MBRT slope was documented up to $\mathrm{OD}=10$, resulting in a range of $10^{2}-10^{7}$ cells for the viable count.
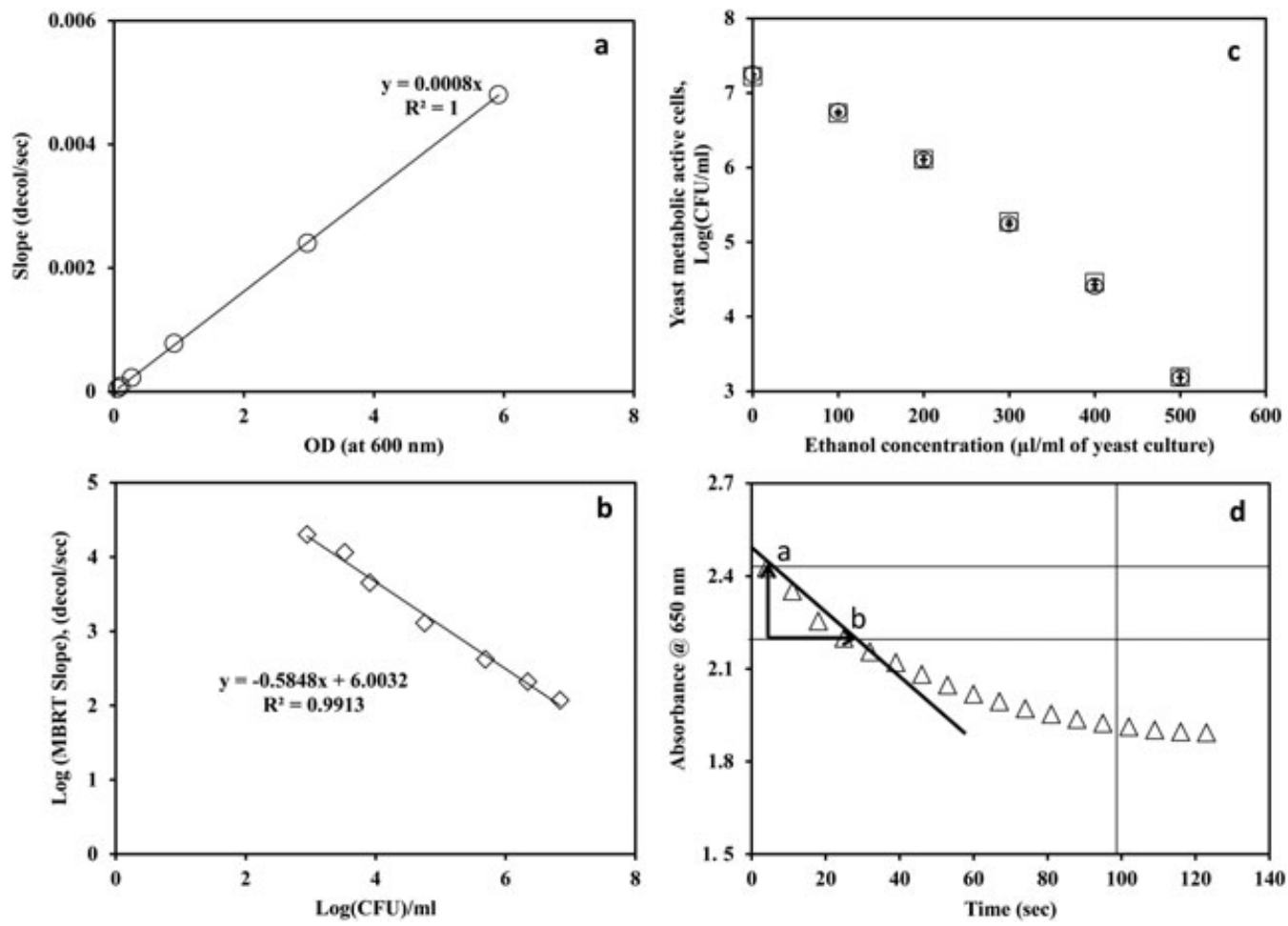

\section{Verifying the MBRT in the robotic set-up}

To validate the correlations observed from the sensitivity analysis, and to establish the applicability of the MBRT, samples from an exponential growing culture were challenged with a range of different concentrations of ethanol (10-50\%), with the purpose of inhibiting/killing portions of the cells. These samples were divided into two fractions, one used for a spread-plate CFU experiment and the other applied for MBRT. By utilizing the previously established relationship between MBRT slopes and metabolically active cells (based on $\mathrm{CFU} / \mathrm{ml}$ ), it was possible to quantify the concentration of metabolically active cells in the samples. Finally, these calculated concentrations were shown to be well correlated with the results generated on the spread plates, validating the methodology. All experiments were carried out in duplicate to establish the robustness of the method through

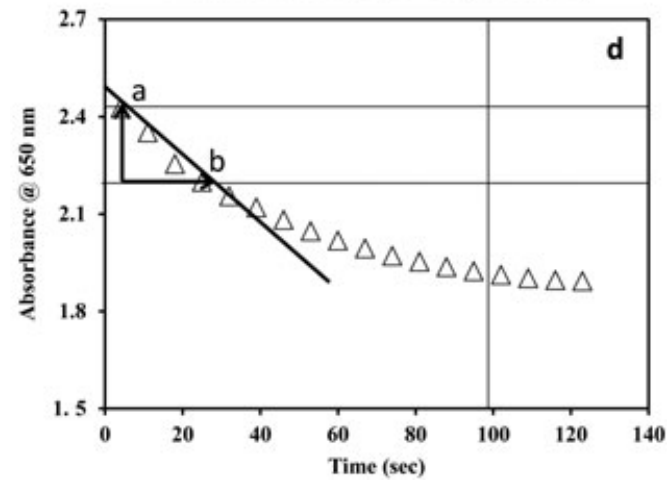

Figure I. MBRT sensitivity and validation. (A) Correlation between OD and MBRT slope: open circles, experimental points; solid line, best fit with $R^{2}=1$; best-fit equation given by $y=0.0008 x$. (B) Correlation between CFU and metabolically active cells calculated from the MBRT slope; in this case, $\log ($ MBRT slope) and $\log (C F U)$ were fitted as a straight line; solid line, best fit with $R^{2}=0.99$, best-fit equation given by $y=-0.5848 x+6.0032$. (C) Inhibition experimental data from ethanol concentration in S. cerevisiae, where open square and open circles describe the CFU from MBRT and from agar plates, respectively. (D) Methylene blue decoloration rates (slopes), recorded as a kinetic measurement at $650 \mathrm{~nm}$ over 2 min at $7 \mathrm{~s}$ intervals; MBRT was determined from the slope in the linear phase 
evaluation of the standard deviations (SDs), as illustrated in Figure 1c. Figure 1d shows the time profile of the methylene blue decoloration at $650 \mathrm{~nm}$ as an output of the kinetic measurements (see Materials and methods).

\section{Assessment of metabolically active cell number by MBRT}

To evaluate the applicability of the method as a tool for physiological characterization, it was tested in a standard physiological characterization of a batch cultivation of $S$. cerevisiae CEN. PK113-7D. Figure 2a displays the actual OD measurements for the full batch characterization (open triangles) plotted with the calculated $\mathrm{OD}$, based on the MBRT (open circles). From this figure it is evident that there was a high degree of correlation between the OD values for the entire exponential growth phase, and it was only as the culture entered the stationary phase that substantial deviations started to occur. These deviations were in fact representative of the drop in metabolic activity observed as the culture progressed into the stationary phase. Since OD measurements are non-

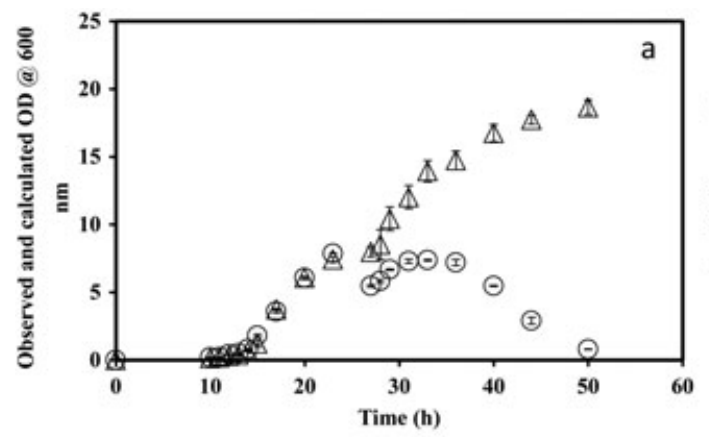

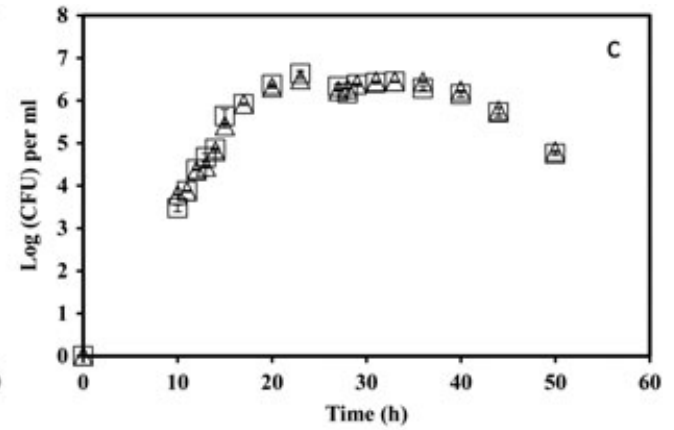

discriminating with regard to the metabolic state of the cells, the calculated ODs based on the MBRT measurements are actually a better physiological tool for characterizing microbial growth phases. This was further evident from Figure 3b, in which the decoloration rates are plotted against time, yielding a growth curve entirely based on only live, metabolically active and dividing cells. Closer investigation of the MBRT-based growth curve in Figure $2 \mathrm{~b}$ further reveals a sharp drop in metabolic activity at $23 \mathrm{~h}$, consistent with the diauxic shift, which is less pronounced in the case of the non-discriminatory OD curve of Figure 2a. Extrapolation of the growth rate from both this curve and the corresponding curve based on OD measurements were in good agreement with the OD-based $\mu_{\max }=0.372 \pm 0.01 / \mathrm{h}$ and the MBRTbased one resulting in $\mu_{\max }=0.365 \pm 0.03 / \mathrm{h}$, both correlating well with the literature (Bakker et al., 2000). Although easily within reasonable SD, the slightly lower growth rate based on the MBRT may be explained by the fact that this measurement does not take the increasing fraction of nonmetabolic active cells into account. In Figure 2c the calculated concentration of metabolically active

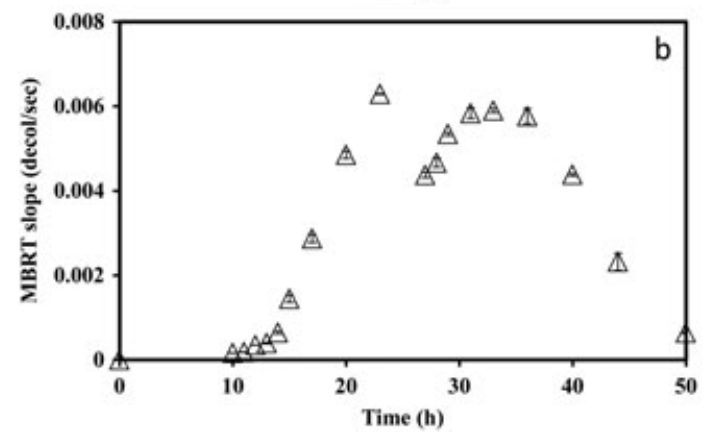

Figure 2. Physiology of S. cerevisiae II3-7D correlated to MBRT slope. (A) Correlation between actual OD (open triangle) and calculated OD from the MBRT slope (open circle). (B) Change of MBRT slope with time (open triangle). (C) Correlation between actual CFU from plate method (open square) with the metabolically active cell count from the MBRT slope (open triangle) 

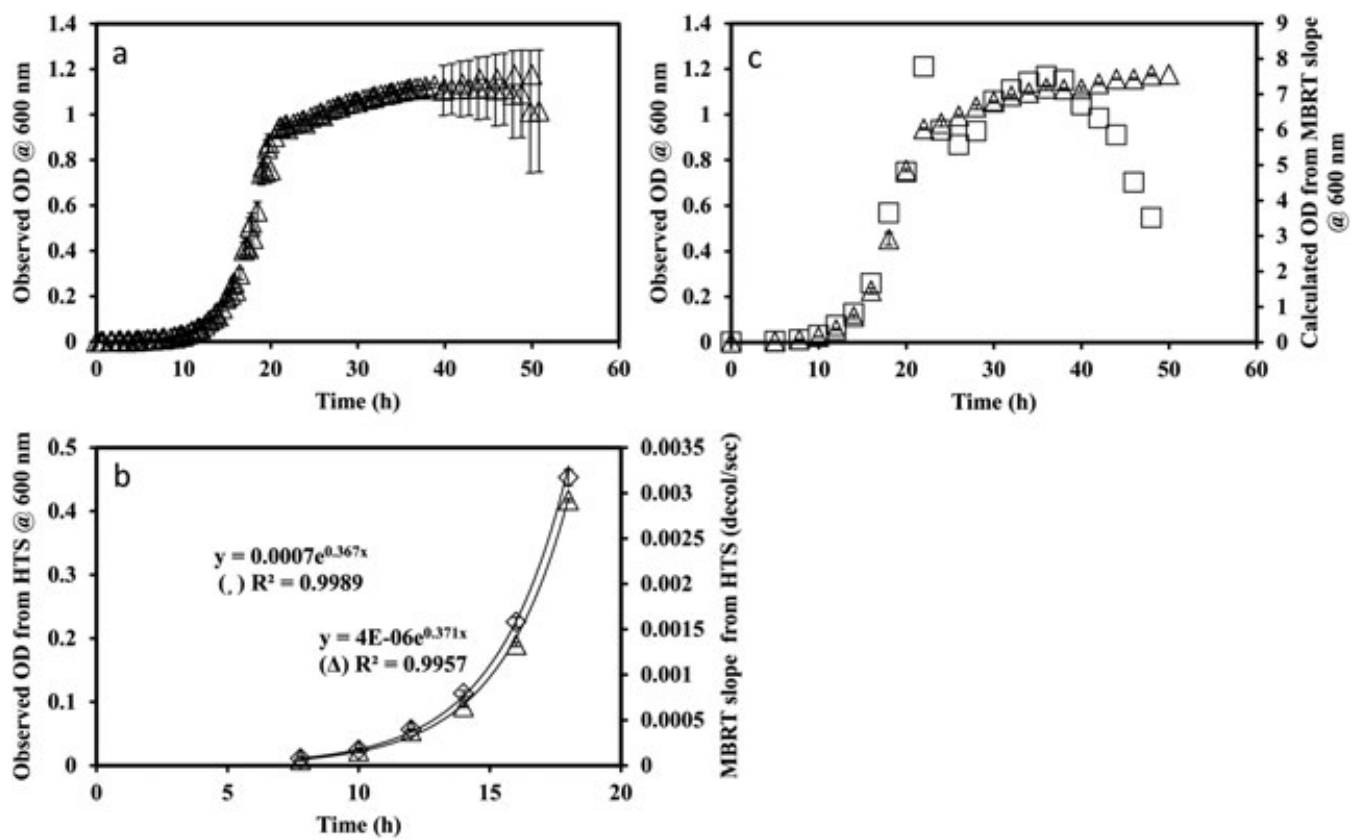

Figure 3. High-throughput physiology data. (A) Overall OD, summarized on a plate-to-plate basis $(n=3)$, with the OD of up to 20 individual wells logged with their respective SDs on a time-point basis. (B) $O D=600$ nm (open diamond) demonstrates the same specific growth rate that was measured from the MBRT slope (open triangle) and it is similar to the maximum specific growth rate from batch cultivation of S. cerevisiae II3-7D. (C) Correlation between actual OD (open triangle) and calculated OD from MBRT slope (open rectangle)

cells is plotted against CFU spread-plate results, proving the method highly accurate, with only minor SDs attached to each individual measurement.

\section{High-throughput scalability study}

The high-throughput, microtitre-based cultivations were evaluated on two levels to ascertain the scalability of the MBRT methodology and to evaluate the applicability of the high-throughput method for growth rate characterization.

Growth rates were calculated by measuring the OD of the individual wells of the microtitre plates over time, thereby treating each well of the microtitre plates as separate biological replicates $(n=49$; data not shown). In addition to this individuallybased calculation strategy, the results on a plate to plate level $(n=3)$ were summarized and plotted as a single growth curve by taking the average optical density of up to 20 well replicates and plotting them for the individual time points at which the plates were measured. This strategy of evaluating the growth rate as a total of all individual wells, but also summarizing all plate results as coherent measurements resulting in a single growth curve, makes it possible to ascertain the robustness of the methodology. This was evaluated using oneway ANOVA for the individual growth rates, evaluated as the three different populations represented by the separate microtitre plates. With a population variance in the range $\sigma^{2}=2.89-5.88 \times 10^{-5}$ and the test displaying no statistically significant differences $[F(2,48)=0.439, p=0.347]$, the methodology was considered robust. The individual well assessment resulted in a maximal growth rate of $\mu_{\max }=0.366 \pm 0.007 / \mathrm{h}$ (Figure 3a), with a total sample variance of $\sigma^{2}=4.38 \times 10^{-5}$. The low variance and the fact that the individual growth rates displayed no significant differences indicates that the growth phase of each individual well follows an almost identical course, which opens up for the possibility for treating individual wells as representative for single batch cultivation.

To test the downscaled MBRT methodology, triplicate samples were automatically harvested at userdesignated time points and treated with methylene blue, in accordance with the described method, before the decoloration rates were recorded. In 
Figure $3 \mathrm{~b}$ the decoloration rate vs sample time point are plotted, together with the OD at the sample time point. Extrapolation of the growth rates from both the MBRT and OD curves are in good agreement with the OD-based $\mu_{\max }=0.367 \pm 0.009 / \mathrm{h}$ and the MBRT-based one resulting in $\mu_{\max }=0.371 \pm 0.007 /$ $\mathrm{h}$ (Figure 3b). Although in overall good correlation with both literature and bioreactor data, a higher degree of evaporation must be expected, which could affect growth physiology. With evaporation not accounting for more than approximately $5 \%$ in the presented case, the results were not substantially affected and evaporation was disregarded. However, for slower-growing microorganisms, including more in-depth physiological analysis, evaporation becomes a factor that should be taken into account.

\section{Conclusions}

This study was an extension of previous work on prokaryotes, and in part yeast, using methylene blue reduction to gauge metabolic activity in cells in order to correlate CFU with OD. In this study we have shown that the method could be efficiently extended to also include eukaryotes and that, with few modifications, it is adaptable for high-throughput systems. This modified method allows for fast and automatic assessment of the physiological state of the culture and, due to the discrimination between metabolically active and inactive cells, a superior method for estimating bioprocess-relevant growth rates. In addition, the accurate quantification of metabolically active cells in a culture makes it a good alternative to the more laborious and time-requiring method of colony counting. The method could further be adapted for antimicrobial screens that are commonly carried out in microtitre plates. Here, this method would offer a significantly cheaper alternative to many of the staining procedures applied with regard to time, manpower and running costs.

\section{Acknowledgements}

The work was funded by the Danish Research Agency for Technology and Production (Grant No. 09-064967), which partly financed P.B.K., J.T. and A.E.L. and the ERA-NET Industrial Biotechnology Project IPCRES (funding to P.B.K.).
The authors acknowledge the Technical University of Denmark Fermentation Platform at the Department of Systems Biology for providing access to both the Hamilton Microlab STAR liquid handling workstation and bioreactor equipment.

\section{Author contributions}

S.K.N. and P.B.K. contributed equally to the manuscript. S.K.N. performed all experiments and drafted the manuscript; P.B.K. designed the methods applied for the high-throughput set-up and co-wrote the manuscript; A.R. programmed the methods for the high-throughput process; A. E.L. and J.T. discussed the design of experiments; and J.T. and M.W. supervised the work and assisted in the manuscript writing.

\section{Conflict of Interest}

The authors declare no conflicts of interest.

\section{References}

Atherton HV, Newlander JA. 1977. Chemistry and Testing of Dairy Products, 4th edn. AVI: Westport.

Bakker BM, Bro C, Kotter P, et al. 2000. The mitochondrial alcohol dehydrogenase Adh3p is involved in a redox shuttle in Saccharomyces cerevisiae. J Bacteriol 182: 4730-4737.

Bapat P, Nandy SK, Wangikar P, Venkatesh KV. 2006. Quantification of metabolically active biomass using methylene blue dye reduction test (MBRT): measurement of CFU in about $200 \mathrm{~s}$. J Microbiol Methods 65: 107-116.

Davey HM, Kell DB. 1996. Flow cytometry and cell sorting of heterogeneous microbial populations: the importance of single cell analyses. Microbiol Rev 60: 641-696.

Fischer M, Sawers RG. 2013. A universally applicable and rapid method for measuring the growth of Streptomyces and other filamentous microorganisms by methylene blue adsorptiondesorption. Appl Environ Microbiol 79: 4499-4502.

Holm DK, Petersen LM, Klitgaard A, et al. 2014. Molecular and chemical characterization of the biosynthesis of the 6-MSA-derived meroterpenoid yanuthone D in Aspergillus niger. Chem Biol 21: 519-29.

Kaprelyants AS, Gottschal JC, Kell DB. 1993. Dormancy in nonsporulating bacteria. FEMS Microbiol Rev 104: 271-286.

Karner M, Fuhrman JA. 1997. Determination of active marine bacterioplankton: a comparison of universal 16S rRNA probes, autoradiography and nucleoid staining. Appl Environ Microbiol 63: $1208-1213$.

Kell DB, Kaprelyants AS, Weichart DH, et al. 1998. Viability and activity in readily culturable bacteria: a review and discussion of the practical issues. Antonie Van Leeuwenhoek 73: $169-187$. 


\section{S. K. Nandy et al.}

Learoyd SA, Kroll RG, Thurston CF. 1992. An investigation of dye reduction by food-borne bacteria. J Appl Bacteriol 72: 479-485.

Li W, Gou Z, Zhang L, Ding, Z. 2011. A novel and rapid method for yeast vitality evaluation based on the methylene blue dye reduction test. J Am Soc Brew Chem 69: 44-49.

Pettipher GL, Mansell R, McKinnon CH, Cousins C. 1980. Rapid membrane filtration epifluorescent microscopy technique for direct enumeration of bacteria in raw milk. Appl Environ Microbiol 39: $423-429$.
Stentelaire C, Antoine N, Cabrol C, et al. 2001. Development of a rapid and highly sensitive biochemical method for the measurement of fungal spore viability. An alternative to the CFU method. Enzyme Microb Technol 29: 560-566.

Vaija J, Ghommidh C, Navarro JM. 1993. Zymomonas mobilis cell viability: measurement method comparison. Antonie Van Leeuwenhoek 64: 57-66.

Venkateshwaran K, Hattori N, La Duc MT, Kern R. 2003. ATP as a biomarker of viable microorganisms in clean-room facilities. J Microbiol Methods 52: 367-377. 